PAPER

\title{
White matter hyperintensities as a predictor of neuropsychological deficits post-stroke
}

\author{
H Jokinen, H Kalska, R Mäntylä, R Ylikoski, M Hietanen, T Pohjasvaara, M Kaste, T Erkinjuntti
}

See Editorial Commentary, p 1185

J Neurol Neurosurg Psychiatry 2005;76:1229-1233. doi: 10.1136/jnnp.2004.055657

See end of article for authors' affiliations ......................

Correspondence to: Dr H Jokinen, Department of Neurology, Helsinki University Central Hospital, P.O. Box 302, FIN-00029 HUS, Helsinki, Finland; hanna.jokinen@ helsinki.fi

Received 6 October 2004 In revised form

3 February 2005

Accepted 3 February 2005
Objectives: Cerebral white matter hyperintensities (WMHs) on magnetic resonance imaging (MRI) are a recognised risk factor for post-stroke dementia. Their specific relations to cognitive impairment are still not well known. The purpose of this study was to explore how the severity and location of WMHs predict neuropsychological test performance in the context of other brain lesions in elderly stroke patients. Methods: In the Helsinki Stroke Aging Memory Study, 323 patients, aged from 55 to 85 years, completed a detailed neuropsychological test battery and MRI 3 months after an ischaemic stroke. The demographic and MRI predictors of cognition were studied with sequential linear regression analyses.

Results: After age, education and total infarct volume were controlled for, the overall degree of WMHs predicted poor performance in tests of mental speed, executive functions, memory, and visuospatial functions, but not in those of short term memory storage or verbal conceptualisation. However, the contribution of separate white matter regions was relatively low. Only the lesions along the bodies of lateral ventricles were independently associated with speed and executive measures. Additionally, general cortical atrophy clearly predicted a wide range of cognitive deficits while infarct volume had less relevance. Further analyses revealed that executive functions act as a strong mediator between the relationship of WMHs to memory and visuospatial functions.

Conclusions: The degree of WMHs is independently related to post-stroke cognitive decline. The most affected cognitive domains seem to be executive functions and speed of mental processing, which may lead to secondary deficits of memory and visuospatial functions.

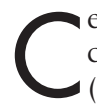
erebral white matter hyperintensities (WMHs) are a common finding on magnetic resonance imaging (MRI) in patients with cerebrovascular diseases and also in healthy elderly persons. Their frequency is correlated with increasing age, vascular risk factors, ${ }^{12}$ cognitive impairment, ${ }^{2}{ }^{3}$ and dementia. ${ }^{4}$ Several studies with neurologically healthy individuals have shown that WMHs, especially around the periventricular area, are related to deficits in speed of cognitive processing and attention. ${ }^{5-7}$ It has been suggested that executive functions mediated by the frontal lobe are particularly vulnerable to the effects of WMHs. ${ }^{89}$ The underlying cause for the observed relationship is assumed to lie in a disruption of the frontal subcortical circuits, which compromises the integrity of the frontal lobe functions. ${ }^{10-12}$ Nevertheless, the clinical relevance of the location of WMHs is still not well known.

Along with the population based studies, much of the literature on white matter has centred on memory clinic samples and dementia outpatients. A central feature of vascular cognitive impairment and dementia with subcortical small vessel pathology seems to be an impairment of executive functioning. ${ }^{13}{ }^{14}$ Previous studies have also indicated that WMHs are an important risk factor for post-stroke dementia, ${ }^{15}$ together with complex interactions of infarct features and brain atrophy. ${ }^{16}$ Despite the fact that WMHs are commonly found in stroke patients, studies investigating their specific role in cognitive performance in this population are few. Recently, Burton and co-workers ${ }^{17}$ suggested that in older stroke patients the volume of WMHs, particularly in the frontal regions, is related to diminished cognitive processing speed and attention, while the volume of temporal lobe hyperintensities is associated with memory impairment. In addition, Sachdev and colleagues ${ }^{18}$ have reported a correlation between white matter pathology and cognitive dysfunction in patients with stroke or transient ischaemic attack.
The purpose of this study was to explore how the severity and location of WMHs predict performance in neuropsychological tests in elderly stroke patients. In our large stroke cohort, we earlier demonstrated that WMHs are associated with executive dysfunction. ${ }^{19}$ In this study, we aimed to investigate in further detail the role of WMHs in executive deficits and mental slowing, and the mediating effects of these deficits to other cognitive functions. Periventricular hyperintensities (PVHs) were analysed separately around both frontal (PVH-FH) and occipital horns (PVH-OH), and along the bodies of the lateral ventricles (PVH-B). In addition, the non-periventricular WMHs in deep, watershed, and subcortical areas (WMH-D) were examined. Total infarct volume, general cortical atrophy, and host factors were considered to be covariate predictors of cognition.

\section{METHODS}

Patients and study design

The Helsinki Stroke Aging Memory Study is a prospective cross sectional study of elderly patients with ischaemic stroke. The patients were 55 to 85 years of age and they were recruited consecutively at the emergency unit of the Helsinki University Central Hospital, Helsinki, Finland $(n=486)$. The details of the protocol and cohort have been reported previously. ${ }^{20}$ The patients went through a clinical
Abbreviations: FOME, Fuld Object Memory Evaluation; MRI, magnetic resonance imaging; $\mathrm{PVH}$, periventricular hyperintensity; $\mathrm{PVH}-\mathrm{B}$, white matter hyperintensities along the bodies of lateral ventricles; $\mathrm{PVH}-\mathrm{FH}$, white matter hyperintensities around frontal horns; $\mathrm{PVH}-\mathrm{OH}$, white matter hyperintensities around occipital horns; WAIS-R, Wechsler Adult Intelligence Scale-Revised; WCST, Modified Wisconsin Card Sorting Test; WMH, white matter hyperintensity; WMH-D, white matter hyperintensities in deep, watershed and subcortical white matter areas; WMS, Wechsler Memory Scale; WMS-R, Wechsler Memory Scale- Revised 
assessment, brain MRI, and a comprehensive neuropsychological examination 3 months after the index stroke. In this study, we included patients who succeeded in completing both the neuropsychological test battery and MRI adequately $(n=323)$. The ethics committee of the Department of Neurology, Helsinki University Central Hospital, approved the study, and all patients participating in the study gave an informed consent.

\section{Neuropsychological examination}

The neuropsychological examination was conducted by observers blinded to the neuroradiological data. The test methods are clinically widely established and they were presented according to their standard instructions and scoring. The details have been described in previous reports. ${ }^{21}{ }^{22}$

\section{Mental speed}

Speed of mental processing and attention were assessed with the Trail Making test part $\mathrm{A}^{23}$ and a modified short form of the Stroop test (24 items with four colours), the coloured dots section (Stroop dots). ${ }^{24}{ }^{25}$ In both of these tests, the time taken to complete the task was recorded. No corrections were made for errors.

\section{Executive functions}

The Trail Making test part $\mathrm{B},{ }^{23}$ and the short form of the Stroop test, coloured colour names section (Stroop words), ${ }^{24}{ }^{25}$ were used in evaluating mental flexibility, set shifting, and response inhibition. In both conditions, the time taken to complete the test and the correct responses were recorded. Errors were not corrected. A modified version of the Wisconsin Card Sorting test (WCST) ${ }^{26}$ was used in assessing abstract problem solving and the ability to shift cognitive sets. The total number of correct responses and perseverative errors were recorded. Verbal fluency was measured in letter generation (letter $\mathrm{K}$ ) and semantic category (animals), both in 60 seconds, ${ }^{25}$ and were considered in conjunction with the other executive tests.

\section{Memory functions}

Digit span forward and backward tasks were administered according to the Wechsler Memory Scale (WMS $)^{27}$ in measuring short term storage and working memory. The tasks were included in the final analysis as a sum variable, as they gave identical results. Verbal memory was assessed by using the story $\mathrm{A}$, immediate and delayed recall of the logical memory subtest of the Wechsler Memory Scale-Revised (WMS-R). ${ }^{28}$ Visual memory was examined with the visual reproduction subtest, immediate and delayed recall, of the WMS-R. ${ }^{28}$ Additionally, learning was assessed with the Fuld Object Memory Evaluation (FOME), ${ }^{29}$ in which the variables were the total retrieval of recalled items in five trials and the delayed free recall.

Verbal reasoning and visuospatial functions The Wechsler Adult Intelligence Scale-Revised (WAIS-R) similarities subtest ${ }^{30}$ was used in evaluating verbal conceptualisation and abstract thinking. Visuospatial and constructive skills were assessed with the WAIS-R block design subtest. ${ }^{30}$

\section{Magnetic resonance imaging}

MRI was carried out with a superconducting system operating at $1.0 \mathrm{~T}$ as detailed earlier. ${ }^{1631}$ The number, site, side, and type of the focal lesions were recorded. Lesions approaching the signal characteristics of the cerebrospinal fluid on Tl weighted images and measuring over $3 \mathrm{~mm}$ in diameter were classed as brain infarcts. The size of the lesion was classified into four groups and the average radii were used for brain infarct volume calculations. ${ }^{16}$ The total volume of all infarct lesions was used for the purposes of this study.

White matter hyperintensities were evaluated visually on proton density weighted images in six white matter areas: $(a)$ around the frontal and $(b)$ posterior horns, $(c)$ along the bodies of lateral ventricles, and in $(d)$ deep, $(e)$ watershed, and $(f)$ subcortical white matter. The hyperintensities were classified on the basis of size and shape (for details and illustrations, see Mäntylä et $\left.a l^{31}\right)$. The extent of PVHs was graded according to a four point scale: 0 , absence of PVHs; 1 , small caps or thin lining; 2, large caps or smooth halo; and 3, extending caps or irregular halo. The extent of WMHs in deep; watershed and subcortical areas was graded according to a six point scale: 0 , absence of WMHs; 1 , only small focal lesions; 2, at least one large focal; no confluent lesions; 3, at least one focal confluent; no diffusely confluent lesions; 4 , at least one diffusely confluent lesion; and 5, extensive WMHs. In the present study, we focused on the PVHs around frontal and occipital horns and along the bodies of lateral ventricles separately, and on the other areas combined (the most severe gradus of deep, watershed, and subcortical WMHs). The analyses were performed assuming that the white matter gradings were quantitative interval scales.

Brain atrophy was rated visually from 0 to 3 (none, mild, moderate, severe) by comparison with standard images. Cortical and central brain atrophy was rated separately for both hemispheres based on Tl weighted images. General cortical atrophy was rated in the frontal, parietal, and occipital lobes, and in the temporal neocortex. Medial temporal lobe atrophy was rated on coronal slices in the hippocampal formation and the entorhinal cortex (parahippocampal gyrus). In the present study, the ratings of general cortical atrophy and medial temporal lobe atrophy were all combined into a sum variable (range 0-36). Central atrophy was rated by evaluating the width of the temporal, frontal, and occipital horns, the bodies of lateral ventricles, and the third ventricle. These ratings were also combined into a sum variable (range 0-27) for preliminary descriptive purposes, but they were not included in the analyses because of their multicollinearity with age, cortical atrophy, and white matter ratings.

\section{Statistical analysis}

The descriptive data of the demographic and clinical variables were studied with the $\chi^{2}$ test and Pearson's correlation coefficients. The predictors and mediators of cognitive functioning were investigated with a sequential (hierarchical) linear regression analysis, which allows examination of several sets of predictor variables in a given order. Each predictor variable was analysed when controlling for previously and/or simultaneously entered variables. A similar method has been used previously in studying MRI predictors of cognition..$^{32}$ The neuropsychological measures were used as dependent variables individually, and variables including (a) age and years of education, $(b)$ the total volume of infarcts, (c) WMHs in four target areas, and (d) cortical atrophy, were used as predictor variables in the subsequent steps respectively. The percentage of missing data in the neuropsychological tests varied between 0 and 14\%, with the highest percentage in variables of the Trail Making test B, WCST, and FOME. The missing values were not imputed. Because of multiple analyses, $\mathrm{p}<0.01$ was applied to test statistical significance in order to minimise the possibility of type I error.

\section{RESULTS}

Characteristics and MRI findings of the study sample The mean (SD) age of the patients was 70.3 (7.6) years, and of the 323 patients, $160(49.5 \%)$ were men. On the average, 
they had 9.5 (4.2) years of education. The mean (SD) result of the Mini Mental State Examination ${ }^{33}$ was 26.3 (3.2); range 14-30. The diagnostic criteria of dementia according to the DSM-III-R ${ }^{34}$ were fulfilled with 47 (14.6\%) of the patients.

The frequencies of MRI ratings for WMHs are presented in table 1. As could be expected, most of the MRI findings correlated significantly with age and with each other (data not shown), but sex was not associated with MRI findings. On average, patients had 3.1 (2.3) brain infarcts, with an estimated total volume of $25.7(39.8) \mathrm{cm}^{3}$. The mean (SD) of the cortical atrophy ratings was 14.3 (8.7) (range 0-36), and that of central atrophy ratings was 11.4 (8.5) (range 0-27).

\section{Predictors of neuropsychological functioning}

The results of the sequential regression analyses, showing the relationships of the demographic and MRI predictors to the neuropsychological tests, are given in table 2. At each step, the explanatory power of the model accumulates as additional sets of predictor variables are entered. Thus, as an example, the highest increase in explained variance in Trail Making B time was produced by the demographic factors (step 1) and WMHs (step 3).

At the first step, the subject's age and years of education were entered into the regression model. The step, as a whole, predicted all the neuropsychological tests at the $\mathrm{p}<0.001$ level. At the second step, the estimated total volume of infarcts was added to the model and was found to be significant for the Trail Making A time and B correct responses, Stroop dots, verbal fluency (animals), WMS-R visual reproduction, and WAIS-R block design.

Independently of the demographic factors and infarct volume, the third step, including the WMHs measured in four target regions, significantly predicted Trail Making A and B time, Stroop dots and words, WCST correct responses, verbal fluency (animals), WMS-R visual reproduction, FOME delayed recall, and WAIS-R block design. Nevertheless, the contribution of hyperintensities in single white matter regions was found to be relatively weak, which was probably affected in part by their strong mutual correlations. Only PVH-B independently predicted Trail Making A time (standardised $\beta$ coefficient $0.245, p=0.002$ ) and WCST correct responses $(\beta=-0.238, p=0.007)$. The analyses were also performed by using all the six white matter regions as separate variables (deep, watershed, and subcortical regions together with the three periventricular regions). The results remained substantially unchanged, and no specific associations were found between the non-periventricular regions and cognitive performance.

At the last step, cortical atrophy was added to the model and was found to have an independent association with several variables of the Trail Making, Stroop, WCST, verbal

Table 1 Regional frequency distributions (percentages) of WMHs

\begin{tabular}{lcccc}
\hline Grade & $\begin{array}{l}\text { PVH-FH, } \\
\text { range 0-3 }\end{array}$ & $\begin{array}{l}\text { PVH-B, } \\
\text { range 0-3 }\end{array}$ & $\begin{array}{l}\text { PVH-OH, } \\
\text { range 0-3 }\end{array}$ & $\begin{array}{l}\text { WMH-D, } \\
\text { range 0-5 }\end{array}$ \\
\hline 0 & $5(1.5)$ & $69(21.4)$ & $94(29.1)$ & $16(5.0)$ \\
1 & $181(56.0)$ & $142(44.0)$ & $47(14.6)$ & $100(31.0)$ \\
2 & $96(29.7)$ & $63(19.5)$ & $33(10.2)$ & $75(23.2)$ \\
3 & $41(12.7)$ & $49(15.2)$ & $149(46.1)$ & $85(26.3)$ \\
4 & - & - & - & $33(10.2)$ \\
5 & - & - & - & $14(4.3)$ \\
\hline
\end{tabular}

$\mathrm{PVH}-\mathrm{B}$, white matter hyperintensities along the bodies of lateral ventricles; $\mathrm{PVH}-\mathrm{FH}$, white matter hyperintensities around frontal horns; $\mathrm{PVH}-\mathrm{OH}$, white matter hyperintensities around occipital horns; WMH, white matter hyperintensity; WMH-D, white matter hyperintensities in deep, watershed, and subcortical white matter areas fluency, WMS-R logical memory and visual reproduction, FOME, and WAIS-R block design tests.

All the significant associations were in the expected direction. Because of substantial correlations between the predictor variables, relatively high multicollinearity was observed. This can be assumed to be a typical feature of the study population.

\section{Executive functions and speed as mediators for other cognitive deficits}

As WMHs were associated with measures of executive functions and mental speed as well as memory and visuospatial functions, we tested whether there are mediating effects between these relationships. Composite scores were constituted from the standardised $\mathrm{z}$ scores of the measures of executive functions (Trail Making B-A subtraction score and B correct responses, Stroop words-dots subtraction score and words correct responses, verbal fluency, and WCST), mental speed (Trail Making A and Stroop dots) and memory performance (FOME, WMS-R logical memory and visual reproduction). WMHs were here considered as a sum variable constituted from the four regional white matter variables. WMH-D was first recoded into a four point scale corresponding to the PVH scales, and therefore the range of the sum variable was 0 to 12 .

As analysed with linear regression analysis, WMHs predicted the memory score significantly (standardised

Table 2 Sequential linear regression analyses with neuropsychological tests handled individually as dependent variables

\begin{tabular}{|c|c|c|c|c|}
\hline \multirow[b]{3}{*}{$\begin{array}{l}\text { Dependent } \\
\text { variables }\end{array}$} & \multicolumn{4}{|c|}{ Predictor variables } \\
\hline & Step 1 & Step 2 & Step 3 & Step 4 \\
\hline & $\begin{array}{l}\text { Age and } \\
\text { education }\end{array}$ & $\begin{array}{l}\text { Total } \\
\text { infarct } \\
\text { volume }\end{array}$ & $\begin{array}{l}\text { WMHs } \\
\text { in four } \\
\text { target } \\
\text { regions }\end{array}$ & $\begin{array}{l}\text { Cortical } \\
\text { atrophy }\end{array}$ \\
\hline \multicolumn{5}{|l|}{ Trail Making } \\
\hline A, time & $0.179 \ddagger$ & 0. $254 \ddagger$ & $0.313 \ddagger$ & $0.350 \ddagger$ \\
\hline B, time & $0.111 \ddagger$ & 0.120 & $0.202 \ddagger$ & 0.211 \\
\hline B, correct & $0.107 \ddagger$ & $0.155 \ddagger$ & 0.185 & $0.207 \dagger$ \\
\hline \multicolumn{5}{|l|}{ Stroop } \\
\hline Dots, time & $0.173 \ddagger$ & 0. $202 \ddagger$ & $0.237 t$ & $0.264 \ddagger$ \\
\hline Words, time & $0.056 \ddagger$ & 0.066 & $0.124 \ddagger$ & 0.137 \\
\hline Words, correct & $0.142 \ddagger$ & 0.150 & $0.200 \dagger$ & $0.247 \ddagger$ \\
\hline \multicolumn{5}{|l|}{ WCST } \\
\hline Correct responses & $0.123 \ddagger$ & 0.139 & $0.187 \dagger$ & 0. 201 \\
\hline $\begin{array}{l}\text { Perseverations } \\
\text { Verbal fluency }\end{array}$ & $0.063 \ddagger$ & 0.066 & 0.081 & $0.126 \ddagger$ \\
\hline Letter & $0.073 \ddagger$ & 0.083 & 0.117 & 0.127 \\
\hline Animals & $0.091 \ddagger$ & $0.110+$ & $0.175 \ddagger$ & $0.214 \ddagger$ \\
\hline WMS, digit span & $0.065 \ddagger$ & 0.080 & 0.100 & 0.114 \\
\hline \multicolumn{5}{|c|}{ WMS-R, logical memory } \\
\hline Immediate & $0.049 \ddagger$ & 0. 052 & 0.061 & 0.075 \\
\hline Delayed & $0.068 \ddagger$ & 0.069 & 0.084 & $0.113 t$ \\
\hline \multicolumn{5}{|c|}{ WMS-R, visual reproduction } \\
\hline Immediate & $0.193 \ddagger$ & $0.262 \ddagger$ & $0.323 \ddagger$ & $0.368 \ddagger$ \\
\hline Delayed & $0.188 \ddagger$ & $0.229 \ddagger$ & $0.274 \ddagger$ & $0.317 \ddagger$ \\
\hline \multicolumn{5}{|l|}{ FOME } \\
\hline Total retrieval & $0.090 \ddagger$ & 0.090 & 0.130 & $0.169 \ddagger$ \\
\hline Delayed recall & $0.053 \ddagger$ & 0.058 & $0.107 \dagger$ & $0.141 \ddagger$ \\
\hline \multicolumn{5}{|l|}{ WAIS-R } \\
\hline Block design & $0.181 \ddagger$ & $0.267 \ddagger$ & 0. 304† & $0.332 \ddagger$ \\
\hline Similarities & $0.154 \ddagger$ & 0.155 & 0.178 & 0.186 \\
\hline \multicolumn{5}{|c|}{$\begin{array}{l}\text { The results are expressed as proportions of total variance } \mathrm{R}^{2} \text { explained by } \\
\text { the regression models (cumulative in subsequent steps). Statistical } \\
\text { significance } \mathrm{tp}<0.01, \pm \mathrm{f}<0.001 \text { represents the incremental } \\
\text { explanatory power produced by each step. FOME, Fuld Object Memory } \\
\text { Evaluation; WAIS-R, Wechsler Adult Intelligence Scale-Revised; WCST, } \\
\text { Modified Wisconsin Card Sorting Test; WMH, white matter } \\
\text { hyperintensity; WMS, Wechsler Memory Scale; WMS-R, Wechsler } \\
\text { Memory Scale-Revised }\end{array}$} \\
\hline
\end{tabular}




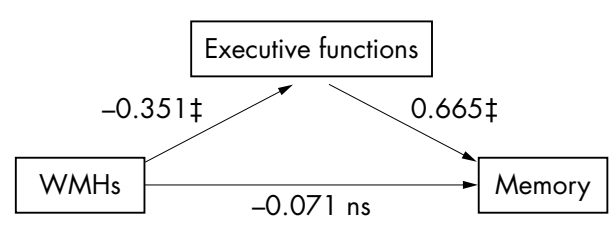

Figure 1 A complete mediation between the relationship of WMHs to memory performance. When executive functions were controlled, WMHs were no longer associated with memory performance. Standardised $\beta$ coefficients of linear regression analyses are presented adjacent to arrows. $\ddagger p<0.001 ; n s$, non-significant.

$\beta=-0.304, p<0.001)$, but when the executive score was added to the model, the predictive value of WMHs dropped substantially, whereas the executive score showed high significance (fig 1). This indicates that executive functions completely mediate the relationship between WMHs and memory performance. ${ }^{35}$ The role of the mediator was also confirmed by the Sobel test, ${ }^{35}$ which proved the pathway to be highly significant $(\mathrm{z}=-5.95, \mathrm{p}<0.0001)$. An identical model was applied to test whether executive functions also explain the association between WMHs and performance in WAIS-R block design. Again, WMHs predicted poor performance in block design as examined with linear regression analysis $(\beta=-0.286, p<0.001)$, but when the executive score was added to the model as another predictor variable, the association declined below the significance level $(\beta=-0.074, p=0.112)$. Therefore, a complete mediating effect was found, and the pathway was confirmed by the Sobel tets $(z=-5.78, p<0.0001)$. In contrast, the mediating effects of mental speed between the relationships of WMHs and other cognitive functions were only partial. When mental speed was controlled, the predictive value of WMHs remained significant for both memory performance $(\beta=-0.155$, $\mathrm{p}<0.01)$ and block design $(\beta=-0.154, p<0.01)$, although indirect effects were indicated by the Sobel tests $(z=-4.98$, $\mathrm{p}<0.0001 ; \mathrm{Z}=-4.93, \mathrm{p}<0.0001)$.

\section{DISCUSSION}

In our stroke cohort, cerebral WMHs were significantly associated with specific cognitive deficits irrespective of age and coexisting brain pathology. After adjusting for age, education, and infarct volume, we discovered that the overall degree of WMHs predicted poor performance in neuropsychological tests measuring speed of mental processing and executive functions. Furthermore, WMHs predicted poor performance in a visual memory test, a delayed recall of object learning, and a visuospatial task. WMHs were not related to short term storage (working memory), story recall, or verbal conceptualisation.

The contribution of hyperintensities in single white matter regions was low compared with the effect of the overall WMHs. Contrary to our expectations, PVH-FH had no specific relevance either to executive functions or other cognitive domains. Significant independent associations were found only between PVH-B and measures of speed and abstract problem solving. The regional white matter measures strongly correlated with each other, which could reduce the possibility of detecting their subtle relative differences. Interestingly, however, the findings parallel a recent study in which WMHs were related to frontal hypometabolism and executive dysfunction regardless of their location. ${ }^{36}$

When the relevance of WMHs to cognitive functions was explored, the variables of age, education, total infarct volume, and general cortical atrophy were considered as covariate predictors of cognition. As could be postulated, age and education together clearly predicted neuropsychological test performance. After these demographic factors were controlled for, infarct volume was only modestly related to some of the measured cognitive functions, such as attention, verbal fluency, visual memory, and visuospatial skills. Instead, cortical atrophy was a strong predictor of a wide range of cognitive domains even after the adjustment for both the demographic factors and other MRI measures.

In previous studies with diverse subject samples, mental or psychomotor speed has been the most commonly affected cognitive domains with regard to WMHs. ${ }^{3-7} 91737$ Our results support the view that white matter damage is an important brain mechanism associated with the slowing of processing speed both in tasks containing a psychomotor component (for example, paper and pencil tests) and in non-motor tasks. Previous studies concerning executive functions have had varying results. Most of the studies have reported correlations between WMHs and executive measures, ${ }^{8} 9323738$ while some have not. ${ }^{17}$ A possible explanation for the inconsistencies may lie in different definitions and operationalisation for the broad concept of executive dysfunction. We defined executive functions as mental flexibility, fluency, abstract problem solving and the ability to shift cognitive sets, and chose to use conventional and well established clinical test methods (Trail Making test, Stroop test, WCST, and verbal fluency test). ${ }^{25}$ The results augment our previous findings ${ }^{19}$ and suggest that in elderly stroke patients WMHs are an independent factor predicting executive deficits.

Consistent with our presumptions, WMHs were also associated with memory performance, namely, visual memory and delayed recall of object learning. On the contrary, we found no significant associations between WMHs and short term storage or story recall. Some of the earlier studies have found correlations with visual ${ }^{8} 9$ and other ${ }^{17}{ }^{32}$ memory functions, but also with working memory. ${ }^{9737}$ An important novel finding was that the significant association between WMHs and memory performance disappeared when it was analysed conditionally to executive functions. The apparent relationship was completely mediated by executive deficits, which therefore suggests that memory deficits may be secondary to executive dysfunction. Moreover, mental speed was a partial mediator between WMHs and memory functions. A possible explanation is that patients with these deficits are unable to fully utilise their mnestic capacity due to inefficient encoding and retrieval strategies, ${ }^{39}$ and slowed mental processing. In our recent report, ${ }^{22}$ we demonstrated that visual memory deficits are in fact associated with medial temporal lobe atrophy irrespective of speed and visuospatial skills. In the present study, WMHs were also related to visuospatial and constructive performance as assessed by the WAIS-R block design subtest. A similar finding has been reported earlier with healthy elderly subjects. ${ }^{40}$ However, in our sample the association was again explained by the mediating role of executive functions.

The strengths of this study are a large and well defined consecutive patient sample and an extensive and clinically relevant neuropsychological test battery. In addition, a careful attempt was made to take into account the versatile brain pathologies typical of elderly stroke patients by using multivariate statistics. As WMHs are strongly correlated with age and with other vascular and degenerative changes, conclusions drawn from simple correlations and bivariate analyses remain debatable. Vascular and degenerative changes may not only coexist but may also be causally related to each other. It should be noted that statistical models encompassing several intercorrelated variables can still be problematic to interpret because of multicollinearity. The possible bias is likely to occur in the conservative direction for the predictor variables that are entered in the model after other correlated variables. Further, it is conceivable that some other factors contributing to cognitive 
impairment are neglected in our analysis. For example, to avoid expanding the analyses excessively, we considered only the total infarct volume as a global measure of stroke and did not take into account the location or the type of infarcts. Even so, we believe that our results are sufficiently robust to answer whether WMHs have an additive contribution to cognitive deficits of elderly stroke patients. Owing to the cross sectional study design, however, any causal conclusions must be viewed with caution.

To conclude, we state that cerebral WMHs have a significant contribution to the neuropsychological performance of elderly patients post-stroke. Executive deficits and slowing of mental processing are the most prominent cognitive characteristics associated with WMHs and these characteristics may lead to secondary impairments of memory and visuospatial functions. Cognitive deficits are best predicted by the overall degree of WMHs, while the role of the distinct white matter regions is weak. In clinical practice, both WMHs and cortical atrophy should be regarded as relevant contributors of vascular cognitive impairment.

\section{ACKNOWLEDGEMENTS}

This study was financially supported by the Finnish Graduate School of Psychology. We wish to thank Professor D J Libon for his helpful comments on the manuscript. We also thank P Keskivaara MA and P Lahti-Nuuttila MA, Department of Psychology, University of Helsinki, for the statistical support and review.

\section{Authors' affiliations}

H Jokinen, Department of Psychology, University of Helsinki and Unit of Neuropsychology, Department of Neurology, Helsinki University Central Hospital, Finland

H Kalska, Department of Psychology, University of Helsinki, Finland

R Mäntylä, Department of Radiology, Helsinki University Central Hospital, Finland

R Ylikoski, M Hietanen, Unit of Neuropsychology, Department of Neurology, Helsinki University Central Hospital, Finland

T Pohjasvaara, Department of Neurology, Lohja Hospital and Memory Research Unit, Department of Neurology, Helsinki University Central

Hospital, Finland

M Kaste, Department of Neurology, Helsinki University Central Hospital, Finland

T Erkinjuntti, Memory Research Unit, Department of Neurology, Helsinki University Central Hospital, Finland

Competing interests: none declared

Ethics approval: The study was approved by the Ethics Committee of the Department of Neurology, Helsinki University Central Hospital, Helsinki, Finland. The study design was first fully explained and written information was provided to the patients. If they agreed to participate, they signed an informed consent.

\section{REFERENCES}

1 Breteler MMB, van Swieten JC, Bots ML, et al. Cerebral white matter lesions, vascular risk factors, and cognitive function in a population-based study: The Rotterdam study. Neurology 1994;44:1246-52.

2 Longstreth WTJ, Manolio TA, Arnold A, et al. Clinical correlates of white matter findings on cranial magnetic resonance imaging of 3301 elderly people: the Cardiovascular Health Study. Stroke 1996;27:1274-82.

3 Junqué $C$, Pujol J, Vendrell $P$, et al. Leuko-araiosis on magnetic resonance imaging and speed of mental processing. Arch Neurol 1990;47:151-6.

4 Wu CC, Mungas D, Petkov Cl, et al. Brain structure and cognition in a community sample of elderly Latinos. Neurology 2002;59:383-91.

5 Fukui T, Sugita K, Sato Y, et al. Cognitive functions in subjects with incidental cerebral hyperintensities. Eur Neurol 1994;34:272-6.

6 Ylikoski R, Ylikoski A, Erkinjuntti T, et al. White matter changes in healthy elderly persons correlate with attention and speed of mental processing. Arch Neurol 1993;50:818-24.
7 de Groot JC, de Leeuw F-E, Oudkerk M, et al. Cerebral white matter lesions and cognitive function: the Rotterdam Scan Study. Ann Neurol 2000;47:145-51.

8 DeCarli C, Murphy DGM, Tranh M, et al. The effect of white matter hyperintensity volume on brain structure, cognitive performance, and cerebral metabolism of glucose in 51 healthy adults. Neurology 1995;45:2077-84.

9 Cohen RA, Paul RH, OH BR, et al. The relationship of subcortical MRI hyperintensities and brain volume to cognitive function in vascular dementia. $J$ Int Neuropsychol Soc 2002;8:743-52.

10 O'Sullivan M, Jones DK, Summers PE, et al. Evidence for cortical "disconnection" as a mechanism of age-related cognitive decline. Neurology 2001;57:632-8.

11 Pugh KG, Lipsitz LA. The microvascular frontal-subcortical syndrome of aging. Neurobiol Aging 2002;23:421-31.

12 Tekin S, Cummings JL. Frontal-subcortical neuronal circuits and clinical neuropsychiatry: An update. J Psychosom Res 2002;53:647-54.

13 O'Brien JT, Erkinjuntti T, Reisberg B, et al. Vascular cognitive impairment. Lancet Neurol 2003;2:89-98.

14 Libon DJ, Bogdanoff B, Leopold N, et al. Neuropsychological profiles associated with subcortical white matter alterations and Parkinson's disease: Implications for the diagnosis of dementia. Arch Clin Neuropsychol 2001;16:19-32.

15 Liu CK, Miller BL, Cummings JL, et al. A quantitative MRI study of vascular dementia. Neurology 1992;42:138-43.

16 Pohjasvaara T, Mäntylä R, Salonen $O$, et al. How complex interactions of ischemic brain infarcts, white matter lesions, and atrophy relate to poststroke dementia. Arch Neurol 2000;57:1295-300.

17 Burton EJ, Kenny RA, O'Brien J, et al. White matter hyperintensities are associated with impairment of memory, attention, and global cognitive performance in older stroke patients. Stroke 2004;35:1270-5.

18 Sachdev PS, Brodaty H, Valenzuela MJ, et al. The neuropsychological profile of vascular cognitive impairment in stroke and TIA patients. Neurology 2004;62:912-19.

19 Vataja R, Pohjasvaara T, Mäntylä R, et al. MRI correlates of executive dysfunction in patients with ischemic stroke. Eur J Neurol 2003;10:625-31.

20 Pohjasvaara T, Erkinjuntti T, Vataja R, et al. Dementia three months after stroke. Baseline frequency and effect of different definitions of dementia in the Helsinki Stroke Aging Memory Study (SAM) cohort. Stroke 1997;28:785-92.

21 Leskelä $M$, Hietanen $M$, Kalska $H$, et al. Executive functions and speed of mental processing in elderly patients with frontal or nonfrontal ischemic stroke. Eur J Neurol 1999;6:653-61.

22 Jokinen H, Kalska H, Ylikoski R, et al. Medial temporal lobe atrophy and memory deficits in elderly stroke patients. Eur J Neurol 2004;1 1:825-32.

23 Reitan RM. Validity of the Trail Making Test as an indicator of organic brain damage. Percept Mot Skills 1958;8:271-6.

24 Perret E. The left frontal lobe of man and the suppression of habitual responses in verbal categorical behavior. Neuropsychologia 1974;12:323-30.

25 Lezak MD. Neuropsychological assessment, 3rd ed. New York: Oxford University Press, 1995

26 Nelson HE. A modified card sorting test sensitive to frontal lobe defects. Cortex 1976:12:313-24.

27 Wechsler D. A standardized memory scale for clinical use. J Psychol 1945;19:87-95.

28 Wechsler D. WMS-R, Wechsler memory scale-revised manual. San Antonio, TX: Psychological Corporation, 1987.

29 Fuld PA. Fuld object memory evaluation. Chicago: Stoelting, 1982

30 Wechsler D. WAIS-R manual. New York: Psychological Corporation, 1981.

31 Mäntylä R, Erkinjuntti T, Salonen $O$, et al. Variable agreement between visual rating scales for white matter hyperintensities on MRI. Stroke 1997;28:1614-23.

32 Mungas D, Jagust WJ, Reed BR, et al. MRI predictors of cognition in subcortical ischemic vascular disease and Alzheimer's disease. Neurology 2001;57:2229-35

33 Folstein MF, Folstein SE, McHugh PR. "Mini-mental state": A practical method for grading the cognitive state of patients for the clinician. J Psychiatr Res 1975; 12:189-98.

34 American Psychiatric Association Committee on Nomenclature and Statistics. Diagnostic and statistical manual of mental disorders (DSM-III-R), 3rd, revised ed. Washington, DC: American Psychiatric Association, 1987

35 Kenny DA. Mediation and moderation. hitp://users.rcn.com/dakenny/ mediate.htm; 2003 (last accessed 5 January 2005).

36 Tullberg $M$, Fletcher E, DeCarli C, et al. White matter lesions impair frontal lobe function regardless of their location. Neurology 2004;63:246-53.

37 Burton E, Ballard C, Stephens S, et al. Hyperintensities and fronto-subcortical atrophy on MRI are substrates of mild cognitive deficit after stroke. Dement Geriatr Cogn Disord 2003;16:113-18.

38 Cook IA, Leuchter AF, Morgan ML, et al. Cognitive and physiologic correlates of subclinical structural brain disease in elderly healthy control subjects. Arch Neurol 2002;59:1612-20

39 Stuss DT, Levine B. Adult clinical neuropsychology: Lessons from Studies of the Frontal Lobes. Annu Rev Psychol 2002;53:401-33.

40 Garde E, Mortensen EL, Krabbe K, et al. Relation between age-related decline in intelligence and cerebral white-matter hyperintensities in healthy octogenarians: a longitudinal study. Lancet 2000;356:628-34. 